\title{
Percutaneous Treatment of Valvular Heart Disease: Well-known Procedures and Innovative Approaches
}

\author{
Áurea J. Chaves
}

$\mathbf{E}^{2}$ xactly 30 years ago, the field of percutaneous treatment of heart valve diseases was born with the publication of a case of pulmonary stenosis treated by balloon valvuloplasty. ${ }^{1}$ We have since witnessed the exponential growth of this subspecialty, with the emergence of countless revolutionary techniques of percutaneous implantation and valve repair.

In this context, this issue of the Revista Brasileira de Cardiologia Invasiva ( $\mathrm{RBCl}$ ) includes two articles that portray the results of well-established techniques as well as innovations in the percutaneous treatment of valvular heart disease in Brazuk. Aguiar Filho et al., from the Instituto de Cardiologia Dante Pazzanese (São Paulo, SP, Brazil), show the evolution of the first 200 patients with mitral stenosis treated by balloon valvotomy from 1987 to 1990 , their management over time, the probability of being free of restenosis after 20 years of evolution, and predictors of lesion recurrence. In a corresponding editorial, Palacios and Silva, from the Massachusetts General Hospital (Boston, USA), draw attention to the importance of the accurate analysis of valve morphology and function to obtain the best procedural results, the differences in clinical profile and outcome in different published series, and the different techniques available. At the end of their article, they reaffirm its role as a first-line treatment for rheumatic mitral stenosis.

Additionally, Brito Jr., from Hospital Albert Einstein (São Paulo, SP, Brazil), and other physicians from the Brazilian Registry of Transcatheter Aortic Valve Implantation share their experience with an alternative route for CoreValve ${ }^{\circledR}$ implantation, i.e., the subclavian artery. This is a substitute route for patients requiring a prosthesis implant who have peripheral vascular disease that prevents the more preferred femoral access. They show the patient inclusion criteria for the alternative access route, the technique and outcome details, and discuss the potential advantages of this route. In their editorial, Ribeiro and Rodés-Cabau, from the Québec Heart Institute (Québec, Canada), discuss a survey on the current use of different routes for transcatheter aortic valve implantation, particularly in studies using the subclavian approach, and cite the situations in which it should not be employed, such as in the presence of a minimum diameter $\leq 6 \mathrm{~mm}$, circumferential calcification, or a previous ipsilateral pacemaker. They also comment on the recommendations to use the subclavian artery in patients with left mammary artery graft patency, which in the past was a formal contraindication to the use of this route for the percutaneous implant of an aortic prosthesis.

This issue also presents a subanalysis of the SPIRIT Women study, the first multicentre registry dedicated to female patients, which has the additional importance of specifically addressing Latin American women. Grinfeld, from the Hospital Italiano de Buenos Aires (Buenos Aires, Argentina), and other investigators in the SPIRIT Women study evaluated the clinical and angiographic profiles and one-year outcomes of patients from a Latin American cohort treated with the XIENCE ${ }^{\mathrm{TM}} \mathrm{V}$ stent. In a related editorial, $\mathrm{Ng}$ and Lansky, from the Yale University Medical Center (New Haven, USA), comment on studies addressing the differences in results between geographical regions and between genders. They discuss the unique opportunity that this study provides to evaluate the outcomes in Latin American women when compared with other women after treatment with the XIENCE ${ }^{\mathrm{TM}} \mathrm{V}$ stent.

Finally, I would like to share with our readers two pieces of news related to the Journal. In a recent consultation with the Coordination for the Improvement of Higher Education Personnel (Coordenação de Aperfeiçoamento de Pessoal de Nível Superior - CAPES), we were informed that the $\mathbf{R B C I}$ has reached the stratum Qualis B3, the same stratum of other long-established journals, such as the Journal of the Brazilian Medical Association (RAMB). This will enable us to give a score of 20 to each published article for which the author's advisor is a post-graduate professor in medicine. This achievement is related to the increasing bibliometric indices achieved by the $\mathbf{R B C I}$, such as the SCImago index, shown in the chart below, compared with the RAMB and with the Brazilian Archives of Cardiology.

The other information is that the complete version of the RBCl will be available in the English language starting with the first issue of 2012 .

A contract has recently been signed with the renowned international publisher Elsevier. These files will be published on the $\mathrm{RBCl}$ website and concomitantly in the SciELO indexing database. The English version of 


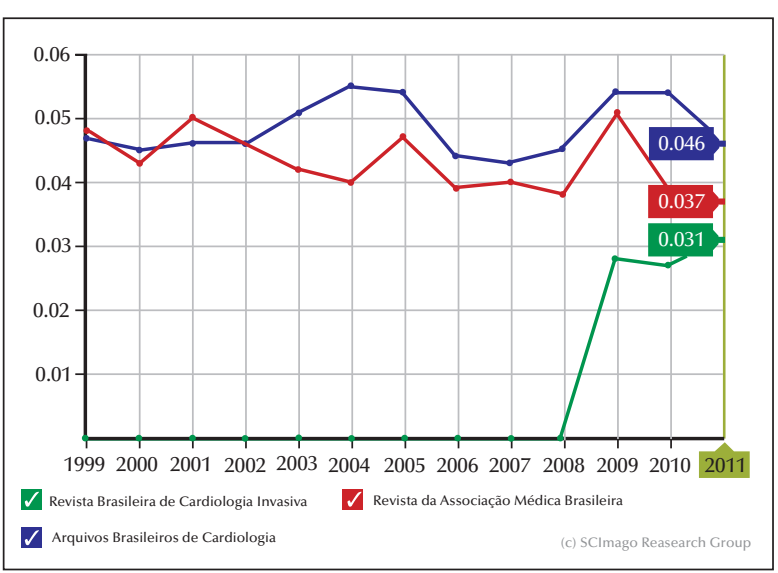

Source: SJR SCImago Journal \& Country Rank. Available at: http://www. scimagojr.com/. Accessed on: Oct. 10, 2012. the RBCl will also be included in Science Direct, the exclusive collection of Elsevier's journals. The quality of the English version is similar to that found in the best journals in the area, and this will certainly promote the dissemination of the $\mathrm{RBCl}$ abroad. This is another important step towards the indexing of the $\mathrm{RBCl}$ in PubMed, our greatest ambition.

Enjoy your reading!

Áurea J. Chaves

Editor

\section{REFERENCE}

1. Kan JS, White RI Jr, Mitchell SE, Gardner TJ. Percutaneous balloon valvuloplasty: a new method for treating congenital pulmonary-valve stenosis. N Engl J Med. 1982;307(9):5402. 